\title{
Overexpression of Aurora-A kinase promotes tumor cell proliferation and inhibits apoptosis in esophageal squamous cell carcinoma cell line
}

\author{
Xiao Xia Wang ${ }^{1,2}$, Rong Liu ${ }^{1}$, Shun Qian Jin ${ }^{1}$, Fei Yue Fan², Qi Min Zhan ${ }^{1}$ \\ ${ }^{I}$ State Key Laboratory of Molecular Oncology, Cancer Institute, Chinese Academy of Medical Sciences and Peking Union Medical \\ College, Beijing 100021, China; ${ }^{2}$ Institute of Radiation Medicine, Chinese Academy of Medical Sciences and Peking Union Medical \\ College, Tianjin 300192, China
}

Aurora-A kinase, a serine/threonine protein kinase, is a potential oncogene. Amplification and overexpression of Aurora-A have been found in several types of human tumors, including esophageal squamous cell carcinoma (ESCC). It has been demonstrated that cells overexpressing Aurora-A are more resistant to cisplatin-induced apoptosis. However, the molecular mechanisms mediating these effects remain largely unknown. In this report, we showed that overexpression of Aurora-A through stable transfection of pEGFP-Aurora-A in human ESCC KYSE150 cells significantly promoted cell proliferation and inhibited cisplatin- or UV irradiation-induced apoptosis. Cleavages of caspase-3 and poly (ADPribose) polymerase (PARP) in Aurora-A overexpressing cells were substantially reduced after cisplatin or UV treatment. Furthermore, we found that silencing of endogenous Aurora-A kinase with siRNA substantially enhanced sensitivity to cisplatin- or UV-induced apoptosis in human ESCC EC9706 cells. In parallel, overexpression of Aurora-A potently upregulated the expression of Bcl-2. Moreover, the knockdown of Bcl-2 by siRNA abrogated the Aurora-A's effect on inhibiting apoptosis. Taken together, these data provide evidence that Aurora-A overexpression promoting cell proliferation and inhibiting apoptosis, suggesting a novel mechanism that is closely related to malignant phenotype and anti-cancer drugs resistance of ESCC cells.

Cell Research (2006) 16:356-366. doi:10.1038/sj.cr.7310046; published online 13 April 2006

Keywords: Aurora-A, apoptosis, caspase-3, PARP, esophageal squamous cell carcinoma (ESCC), siRNA

\section{Introduction}

Esophageal squamous cell carcinoma (ESCC) is one of the most aggressive cancers and the overall prognosis for esophageal cancer patients is poor [1]. One of the reasons for this low survival rate is intrinsically resistant to many clinical therapies like chemotherapy and radiotherapy [2]. Tumor development and progression as well as resistance to clinical treatments result mainly from lack of response to apoptotic stimuli. Apoptosis is a genetically controlled mechanism essential for the maintenance of tissue ho-

Correspondence: Qi Min Zhan ${ }^{1}$, Fei Yue Fan ${ }^{2}$

${ }^{1}$ Tel.: 86-10-87788422; Fax: 86-10-67715058;

E-mail: zhanqimin@chinalab.gov.cn

${ }^{2}$ E-mail:faithyfan@yahoo.com

Received 3 Nov 2005; revised 11 Jan 2006; accepted 22 Feb 2006; published online 13 Apr 2006 meostasis, normal development and suppression of oncogenesis [3]. The induction of apoptosis is found to be a common event for different classes of anti-cancer agents. The efficacy of cancer treatments depends not only on the cellular damage they cause but also on the cell's ability to respond to the damage by inducing apoptotic machinery [4]. Accordingly, in cancer, defects in apoptotic pathways lead to cell lack response to apoptotic stimuli and result in resistance to drugs and radiation.

Aurora, a subfamily of serine/threonine protein kinase, includes Aurora-A, Aurora-B and Aurora-C in vertebrates [5]. Increased attention has now been focused on Aurora-A kinase because of its amplification and overexpression in several types of human tumors, such as breast cancer [6], colorectal cancers [7] and pancreatic cancer [8]. Recently Tanaka [9] and Tong [10] have reported that expression of Aurora-A protein is highly increased in ESCC. Ectopic expression of Aurora-A in murine fibroblasts as well as 
mammary epithelia induces centrosome amplification, aneuploidy, and oncogenic phenotype [11], suggesting that when overexpressed, Aurora-A is a potential oncogene. Recent study indicates that Aurora-A overexpression induces a striking increase in resistance to Taxol-induced apoptosis in HeLa cells [12], also, cells depleted of Aurora-A are sensitive to cisplatin-induced apoptosis in MCF-7 cells, and elevated expression of Aurora-A abolishes this response [13]. Although Aurora-A overexpression has been shown to prevent apoptosis by anti-cancer drugs, however, the molecular mechanisms mediating these effects are largely unknown. In addition, the biological effect of deregulated Aurora-A in ESCC remains to be further defined.

In this report, we transfected an Aurora-A expression vector into ESCC KYSE150 cells and established isogenic cell lines that highly express Aurora-A protein. Then we investigated the role of overexpression of Aurora-A in regulating cell growth and cisplatin-, UV-induced apoptosis. We have found that overexpression of Aurora-A can stimulate cell proliferation and inhibit cisplatin- or UV-induced apoptosis. Small interfering RNA (siRNA)-directed suppression of Aurora-A results in enhanced susceptibility to cisplatin- and UV-mediated apoptosis. In agreement with these observations, overexpression of Aurora-A is found to upregulate the expression of Bcl-2, a potent anti-apoptotic molecule, and inhibition of Bcl-2 by siRNA significantly reduces the Aurora-A's effect on inhibiting apoptosis.

\section{Materials and methods}

\section{Cell culture}

Human ESCC cell lines, which were generously provided by Dr Shimada in Kyoto University, were grown in RPMI 1640 supplemented with $10 \%$ fetal bovine serum (FBS) at $37^{\circ} \mathrm{C}$ under $5 \% \mathrm{CO}_{2}$ and saturated moisture.

\section{Stable transfections and establishment of stable cell lines}

Transfections were performed using $8 \mu$ l of LipofectAMINE 2000 (Invitrogen Corporation) and $15 \mu \mathrm{g}$ of pEGFP-Aurora-A (pEGFPAurora-A was constructed by inserting a $1.2 \mathrm{~kb}$ fragment of human Aurora-A cDNA into the Xho I and EcoR I site of pEGFP-C1) or 15 $\mu \mathrm{g}$ of pEGFP empty vector. $48 \mathrm{~h}$ later, the cells were split at ratios of 1:20 and cultured for 2 weeks in the presence of $400 \mu \mathrm{g} / \mathrm{ml}$ of G418 (Geneticin sulfate, GIBCO). For each transfection, all of the colonies were trypsinized and collected to produce stable cell pools.

\section{Western blot analysis}

Western blot analysis was performed as described [3]. Anti-AuroraA rabbit antibody was obtained from Cell Signaling Technology; anticaspase-3 rabbit antibody, anti-PARP mouse monoclonal and anti-Bcl-2 mouse monoclonal were obtained from Santa Cruz Biotechnology.

\section{$R T-P C R$}

RT-PCR was performed as described [2]. cDNA was amplified using the following Aurora-A primers: 5'-AAT GAT TGA AGG
TCG GAT GC-3' (upstream primer) and 5'-TTC TCT GAG CAT TGG CCT CT- $3^{\prime}$ (downstream primer). PCR was performed for 30 cycles under the conditions of annealing at $58{ }^{\circ} \mathrm{C}(30 \mathrm{~s})$, extension at $72{ }^{\circ} \mathrm{C}(30 \mathrm{~s})$, and denaturing at $94{ }^{\circ} \mathrm{C}(30 \mathrm{~s})$ using a Perkin-Elmer thermocycler.

\section{Immunofluorescence}

Cells were fixed with methanol for $10 \mathrm{~min}$ at $-20^{\circ} \mathrm{C}$. After washing in phosphate-buffered saline (PBS), nuclei were labeled with (4',6'-diamidino-2-phenylindole ) DAPI $(0.1 \mu \mathrm{g} / \mathrm{ml})$ for $15 \mathrm{~min}$ in room temperature. Cells were then examined by fluorescence microscopy (Olympus).

\section{Cell growth curve}

$2 \times 10^{4}$ cells were plated in $60 \mathrm{~mm}$ tissue culture dishes (NUNC) in RPMI 1640 10\% FBS. The cells were trypsinized and manually counted on days $1,3,5,7$ and 9. For each plate, cell count was repeated 3 times to draw the cell growth curve.

\section{Colony formation assay}

$1 \times 10^{3}$ cells were plated in $100 \mathrm{~mm}$ tissue culture dishes. After 14 days, the cells were washed with PBS, fixed with methanol and $0.1 \%$ crystal violet. The colonies were manually counted and then photographed.

\section{3-(4,5-Dimethyl-2-thiazolyl)-2,5-diphenyl-2H-tetrazolium bromide (MTT) assay}

To assess chemosensitivity to cisplatin, $5 \times 10^{3}$ cells cultured for $24 \mathrm{~h}$ in 96-well plates were incubated with different concentrations of cisplatin $(10,15,20 \mu \mathrm{M} / \mathrm{L})$ for $72 \mathrm{~h}$. cells were stained with 100 $\mu 1$ sterile MTT dye $(0.5 \mathrm{mg} / \mathrm{ml}$, Sigma $)$ for $4 \mathrm{~h}$ at $37^{\circ} \mathrm{C}$, then culture medium was removed and $150 \mu 1$ of DMSO was added and thoroughly mixed for $15 \mathrm{~min}$. Spectrometric absorbance at wavelength of $570 \mathrm{~nm}$ was measured on a microplate reader (Bio-Rad). Each group contained five wells. The value of $[A 570$ (cisplatin +$) / A 570$ (cisplatin-) $] \times 100 \%$ indicated the cell survival index.

\section{UV survival assay}

Cells were dispensed in $100 \mathrm{~mm}$ tissue culture dishes at a density of $3 \times 10^{3}$ cells. After $24 \mathrm{~h}$ incubation, they were irradiated with UV $\left(10 \mathrm{~J} / \mathrm{m}^{2}\right.$, UV irradiation was carried out in $254 \mathrm{~nm}$ using germicidal lamps), and the cultures were maintained until the surviving cells formed colonies. The colonies that survived after incubation were then stained with crystal violet, counted, and relative colony numbers were obtained.

\section{DAPI analysis}

Cells were treated with $20 \mu \mathrm{M} / \mathrm{L}$ cisplatin or irradiated with UV $\left(30 \mathrm{~J} / \mathrm{m}^{2}\right)$ and then further incubated for $12 \mathrm{~h}$. Cells were fixed with methanol and stained with $0.1 \mu \mathrm{g} / \mathrm{ml}$ of DAPI. DAPI staining and visualization under a fluorescence microscope showed that cells with condensed or fragmented nuclei were in apoptosis.

\section{Flow cytometry}

Cultures of cells at $70 \%$ confluency were treated with $100 \mu \mathrm{M} / \mathrm{L}$ cisplatin or exposed to UV $\left(45 \mathrm{~J} / \mathrm{m}^{2}\right)$ and then further incubated for $24 \mathrm{~h}$. Flow cytometry was performed as described [12]. Fluorescence-activated cell sorting (FACS) was performed with a Becton Dickinson FACSort apparatus and used to quantitate the apoptotic population based on DNA levels. 
siRNA studies

The siRNA sequence that we used is listed as the following: Aurora-A, AUG CCC UGU CUU ACU GUC A; Bcl-2, GCU GCA CCU GAC GCC CUU C; control siRNA, UUC UCC GAA CGU GUC ACG U. Cells were seeded onto $60 \mathrm{~mm}$ plates for $24 \mathrm{~h}$ and transfected with siRNA or control siRNA for $48 \mathrm{~h}$ using LipofectAMINE 2000 according to manufacturer's instructions. To assess the effect of Aurora-A downregulation on sensitivity of cisplatin, UV-induced apoptosis in EC9706, Aurora-A siRNA-(50 nM/L) or control siRNAtransfected cells were plated in 96-well plates for $24 \mathrm{~h}$ and treated with various concentrations of cisplatin $(5,10,15 \mu \mathrm{M} / \mathrm{L})$ for $72 \mathrm{~h}$. Cell viability was evaluated by MTT assay as described. Cisplatin $(60 \mu \mathrm{M} / \mathrm{L})$ - or UV $\left(45 \mathrm{~J} / \mathrm{m}^{2}\right)$-induced apoptosis was quantified by Flow cytometry assay as described. In addition, to assess the effect of Bcl-2 downregulation on Aurora-A's survival in KYSE150/GFPAur cells, Bcl-2 siRNA-(100 nM/L) or control siRNA-transfected cells were plated in 96-well plates for $24 \mathrm{~h}$ and treated with various concentrations of cisplatin $(10,15,20 \mu \mathrm{M} / \mathrm{L})$ for $72 \mathrm{~h}$. Cell viability was evaluated by MTT assay as described. Cisplatin $(100 \mu \mathrm{M} / \mathrm{L})-$ or $\mathrm{UV}\left(45 \mathrm{~J} / \mathrm{m}^{2}\right)$-induced apoptosis was quantified by Flow cytometry assay as described.

\section{Statistical analysis}

All data represent at least three independent experiments. Statistical comparisons were made using Students't-test. $P<0.05$ were considered to represent a statistically significant difference.

\section{Results}

Generation of Aurora-A overexpressing transfectant clones in KYSE150 cell line

Overexpression of Aurora-A protein in ESCC has previously been reported by us and others $[9,10]$. The expression of Aurora-A using a panel of ESCC cell lines was further investigated by Western-blot analysis. As shown in Figure 1A, all tumor cell lines expressed detectable levels of Aurora-A protein. To explore the possibility that Aurora-A may regulate cell proliferation and cisplatin-, UV-induced apoptosis, KYSE150 cells which had relatively low levels of endogenous Aurora-A expression were stably transfected with an empty vector, $p E G F P$, or pEGFP-Aurora-A, a GFP tagged full length Aurora-A expression vector. Cells were selected with G418 and screened using GFP fluorescence for clones that stably express GFP-Aurora-A protein. The identification of transfected clones was further confirmed by Western blot analysis, RT-PCR and immunofluoresence. Totally, 11 stable GFP-Aurora-A clones were established from the KYSE150 cell line, which expressed fusion protein of GFP-Aurora-A (73 kDa) (Figure 1B). Compared to the relatively low levels of endogenous Aurora-A in parental KYSE150, those isogenic cells expressed at least 2-3 folds more Aurora-A. Then two Aurora-A overexpressing clones and one vector control clone were selected for further analysis. We designated these two clones KYSE150/ GFP-Aur-1 and KYSE150/GFP-Aur-2, respectively, while the vector-transfected clone was termed KYSE150/GFP. Western blot analysis was employed to confirm these two clones. As expected, a $73 \mathrm{kDa}$ band, corresponding to GFPAurora-A, was detected in these two clones (Figure 1C lane 2,3 ), but not in empty vector transfected clone (Figure 1C lane 1). In accordance with this result, as shown in Figure 1D, after electrophoresis of RT-PCR products, there were only weak Aurora-A amplification band in KYSE150/GFP

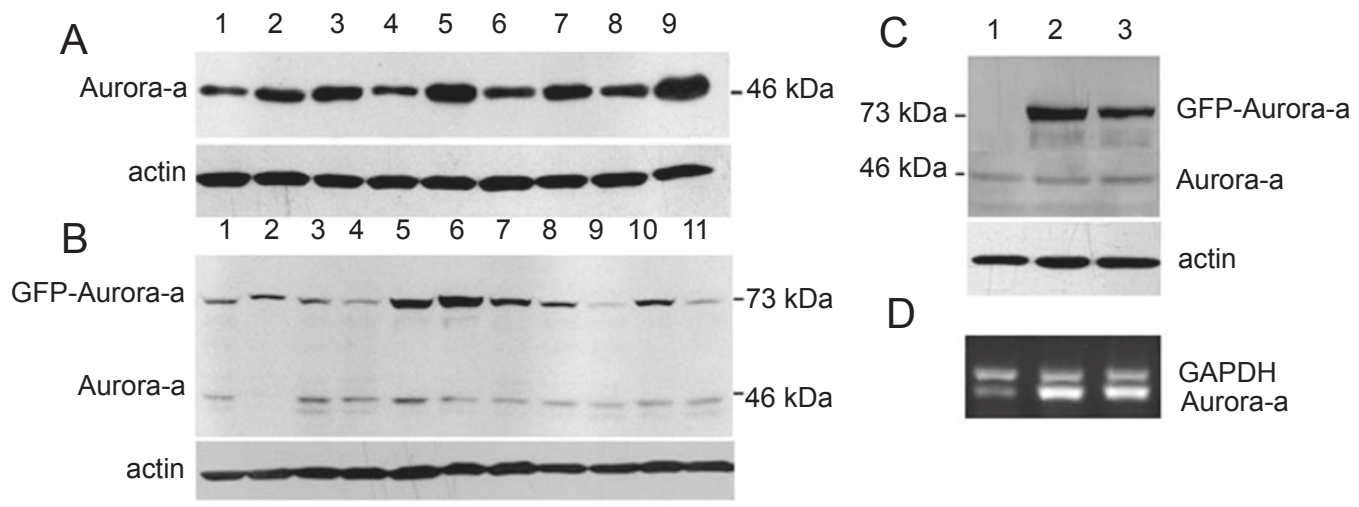

Figure 1 Expression of Aurora-A in ESCC cells lines (A) and stable overexpression of GFP-Aurora-A in KYSE150 cells (B-D). (A) Human ESCC lines (lane1-9: KYSE150, KYSE450, KYSE180, KYSE410, Colo680, KYSE30, KYSE510, KYSE70, EC9706) were grown in RPMI 1640 10\% FBS. $100 \mu \mathrm{g}$ of whole cell protein was used for Western blot analysis with anti-Aurora-A antibody as described. (B) KYSE150 cells were transfected with pEGFP-Aurora-A expressing vector, clones expressing a transfected GFP-Aurora-A protein (lanes 1-11) were detected by Western blot analysis. Because there was a GFP at the N-terminal of Aurora-A, the size of this protein is about $73 \mathrm{kDa}$. (C, D) Two Aurora-A overexpressing clones (lane 2: KYSE150/GFP-Aur-1; lane 3: KYSE150/GFP-Aur-2) and one vector control clone (Lane 1: KYSE150/GFP) were detected by Western blot analysis and RT-PCR. $\beta$-Actin was used as an equal loading control for Western blot and expression of GAPDH was provided to document equivalent loading for RT-PCR. 
cells, and brighter bands in KYSE150/GFP-Aur-1 and KYSE150/GFP-Aur-2 cells.

In addition, Aurora-A overexpressing clones were further confirmed by immunofluorescent assay. As shown in Figure 2A, GFP fused Aurora-A, ectopically expressed, localized to centrosome, mitotic spindle and poles in metaphase and anaphase, whereas in telophase the GFP-Aurora-A primarily mainly co-located at the spindle pole. The Aurora-A staining is consistent with that reported elsewhere [7]. Aurora-A is also a centrosome-associated regulatory molecule and oncogene, the overexpression of which leads to centrosome amplification [11]. As shown in Figure 2B, KYSE150/GFPAur cells contained more than two centrosomes.

\section{Aurora-A overexpression stimulates cell proliferation and colony formation}

We examined the effect of Aurora-A on cell proliferation. Growth curves demonstrated that growth of the KYSE150/
GFP-Aur cells significantly increased compared with the KYSE150/GFP cells (Figure 3A). For the colony formation assay, the rate of colony growth was more obviously increased in the KYSE150/GFP-Aur cells. The colonies from KYSE150/GFP-Aur cells (Figure 3D and E) were much larger than those from the KYSE150/GFP cells (Figure 3C). The numbers of colonies were also much more in KYSE150/GFP-Aur cells compared with the KYSE150/GFP cells (Figure 3B). These results indicated that the Aurora-A overexpressing cells strongly promoted cell proliferation in vitro.

\section{Inhibitory effects of Aurora-A overexpression on cell death}

We next examined if Aurora-A overexpression protected cells from cisplatin and UV-induced cell killing by employing MTT and clonogenic survival assays. When cells were treated with escalating concentration of cisplatin, as

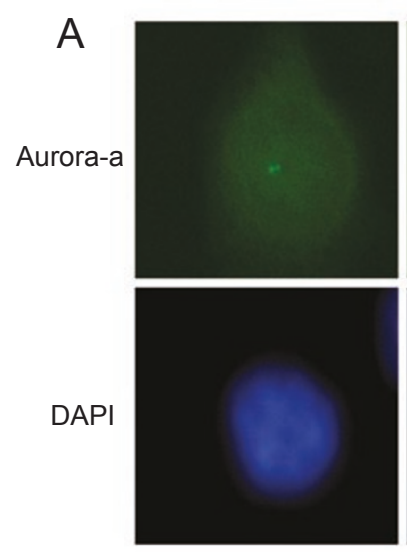

prophase

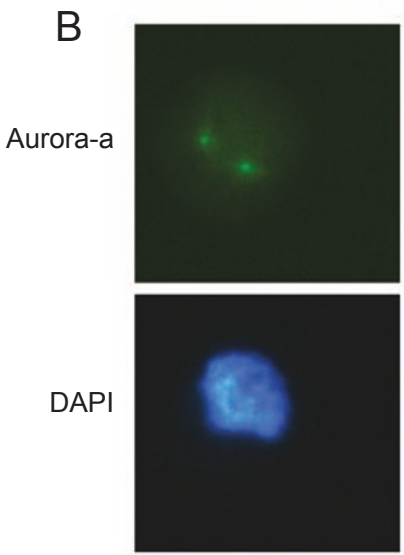

1
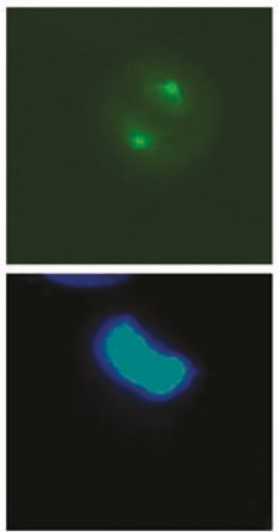

metaphase
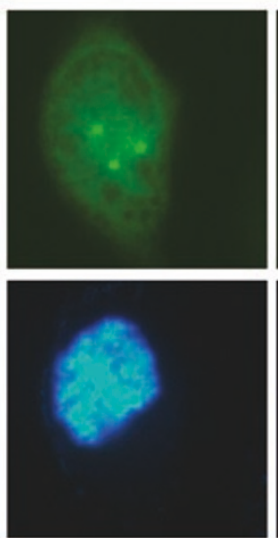

2
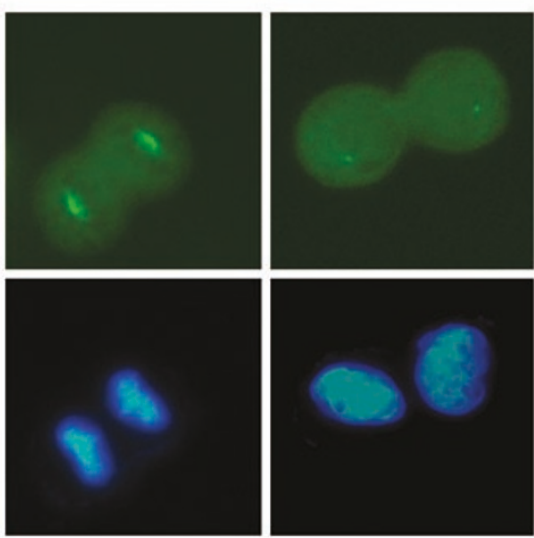

anaphase
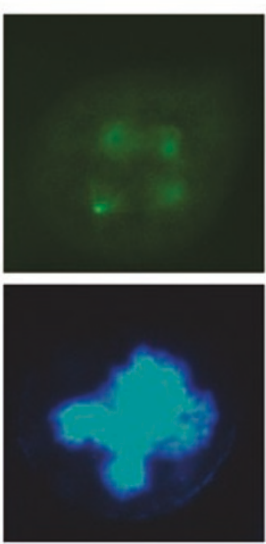

3

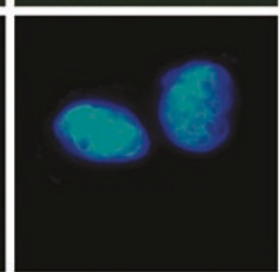

telophase
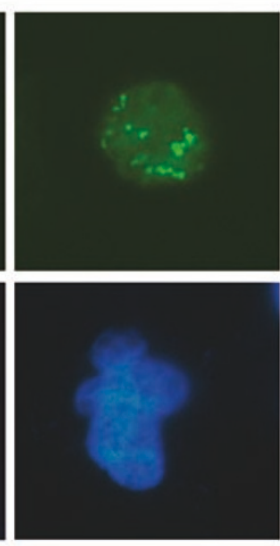

4

Figure 2 Subcellular localization of GFP-fused Aurora-A in human KYSE150 cells. (A) Localization of GFP-Aurora-A mimics distribution of endogenous protein. KYSE150 cells were stable transfected with pEGFP-Aurora-A (green) and stained with DAPI for DNA (blue). Aurora-A is localized to the centrosome, mitotic spindle and poles of metaphase and anaphase cells. (B) Cells have normal numbers of centrosomes (1) and excessive numbers of centrosomes (2-4). 
A

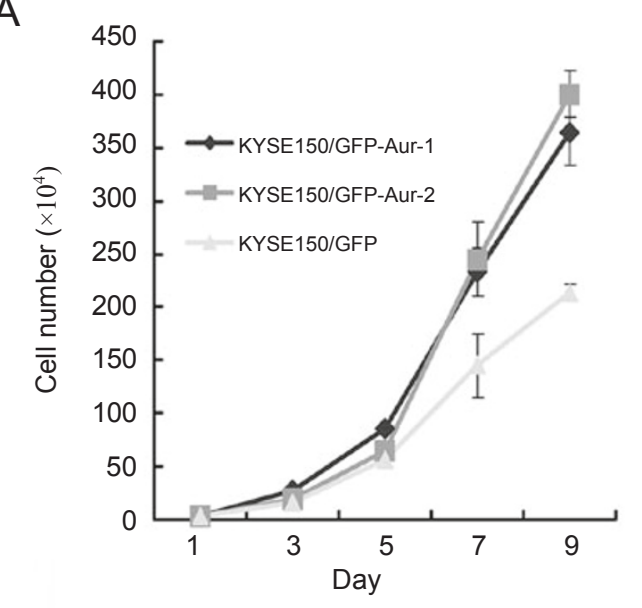

B

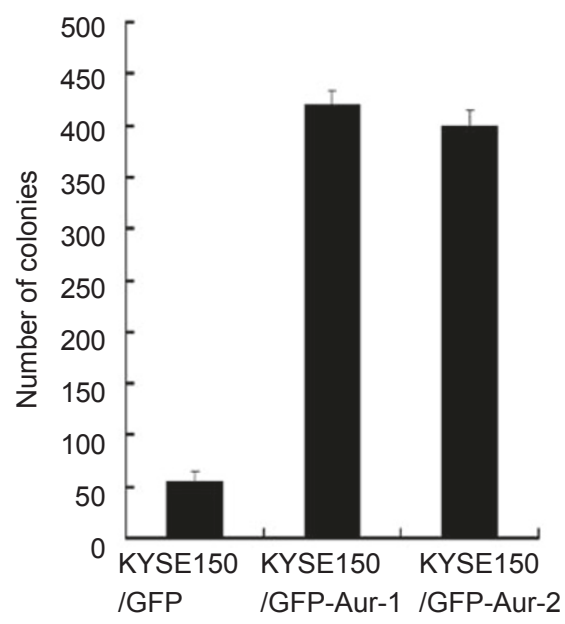

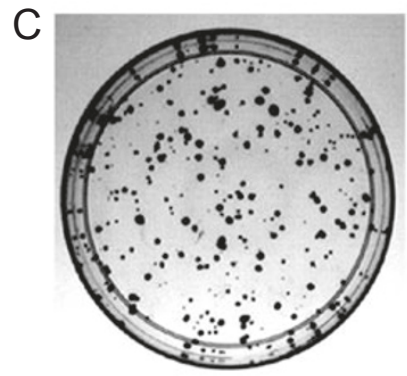

D
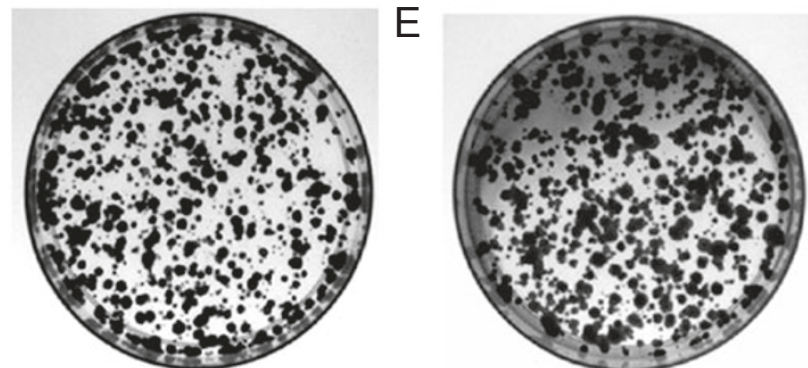

Figure 3 Aurora-A overexpression enhances proliferation, and plating efficiency of KYSE150 cells. (A) Growth curves reveal that KYSE150/GFP-Aur cells proliferate more rapidly than KYSE150/GFP cells. (B-E) Plating efficiency assay reveals changes in colony formation ability. $1 \times 10^{3}$ cells from each of the cell pools were plated in $100 \mathrm{~mm}$ dishes. After 14 days, the colonies were stained with crystal violet, counted, and photographed. (B) Number of colonies counted for each of the three cell pools. Photographs of crystal violet stained KYSE150/GFP (C) KYSE150/GFP-Aur-1 (D), and KYSE150/GFP-Aur-2 (E) colonies. All experiments were performed at least three times with consistent and repeatable results.

shown in Figure 4A, KYSE150/GFP-Aur cells displayed a significantly increased survival, showing $20 \%$ greater than KYSE150/GFP cells. Similarly, cells were exposed to $\mathrm{UV}\left(10 \mathrm{~J} / \mathrm{m}^{2}\right)$, then the percentage of surviving colonies after 10 days was assessed. After UV irradiation, cell survival decreased to about $20 \%$ in KYSE150/GFP cells as determined by the average percentage of colony numbers, whereas more than $92 \%$ and $90 \%$ of the related untreated control cells KYSE150/GFP-Aur-1 and KYSE150/GFPAur-2 cells, respectively, survived (Figure 4B and 4C). These results indicated that overexpression of Aurora-A rendered cells resistant to cisplatin and protected UV-irradiated cells from death.

Overexpression of Aurora-A inhibits cisplatin and $U V$ induced apoptosis

To determine whether increased survival of KYSE150/ GFP-Aur cells after cisplatin and UV treatments was due to Aurora-A inhibition of apoptosis, the morphologic changes in cell nuclei were examined by a fluorescent microscope. When KYSE150/GFP cells were exposed to UV irradiation $\left(30 \mathrm{~J} / \mathrm{m}^{2}\right)$, DAPI-stained KYSE150/GFP cells showed condensed or fragmented nuclei, which is characteristic of apoptosis (Figure 5A-1). Cells incubated for $12 \mathrm{~h}$ after 20 $\mu \mathrm{M} / \mathrm{L}$ cisplatin treatment displayed similar results (data not shown). These results demonstrated that the KYSE150/ GFP cell death was probably due to apoptosis. In contrast, KYSE150/GFP-Aur cells were resistant to cisplatin- and UV-induced cytoxicity, as manifested by little change in cell morphology (Figure 5A-2 and 3). Furthermore, we evaluated the degree of apoptosis by percentage of hypodiploid cells. Cisplatin $(100 \mu \mathrm{M} / \mathrm{L})$ and $\mathrm{UV}\left(45 \mathrm{~J} / \mathrm{m}^{2}\right)$ treatments resulted in higher percentage of KYSE150/GFP cells in sub- $\mathrm{G}_{1}$ phase as compared with KYSE150/GFP-Aur cells (Figure $5 \mathrm{~B}$ and $5 \mathrm{C})$. The apoptotic portion $\left(\mathrm{sub}-\mathrm{G}_{1}\right)$ of KYSE150/GFP-Aur cells was decreased 1.5 folds, compared to the KYSE150/GFP cells. These results indicated that the degree of apoptosis in KYSE150/GFP-Aur cells 

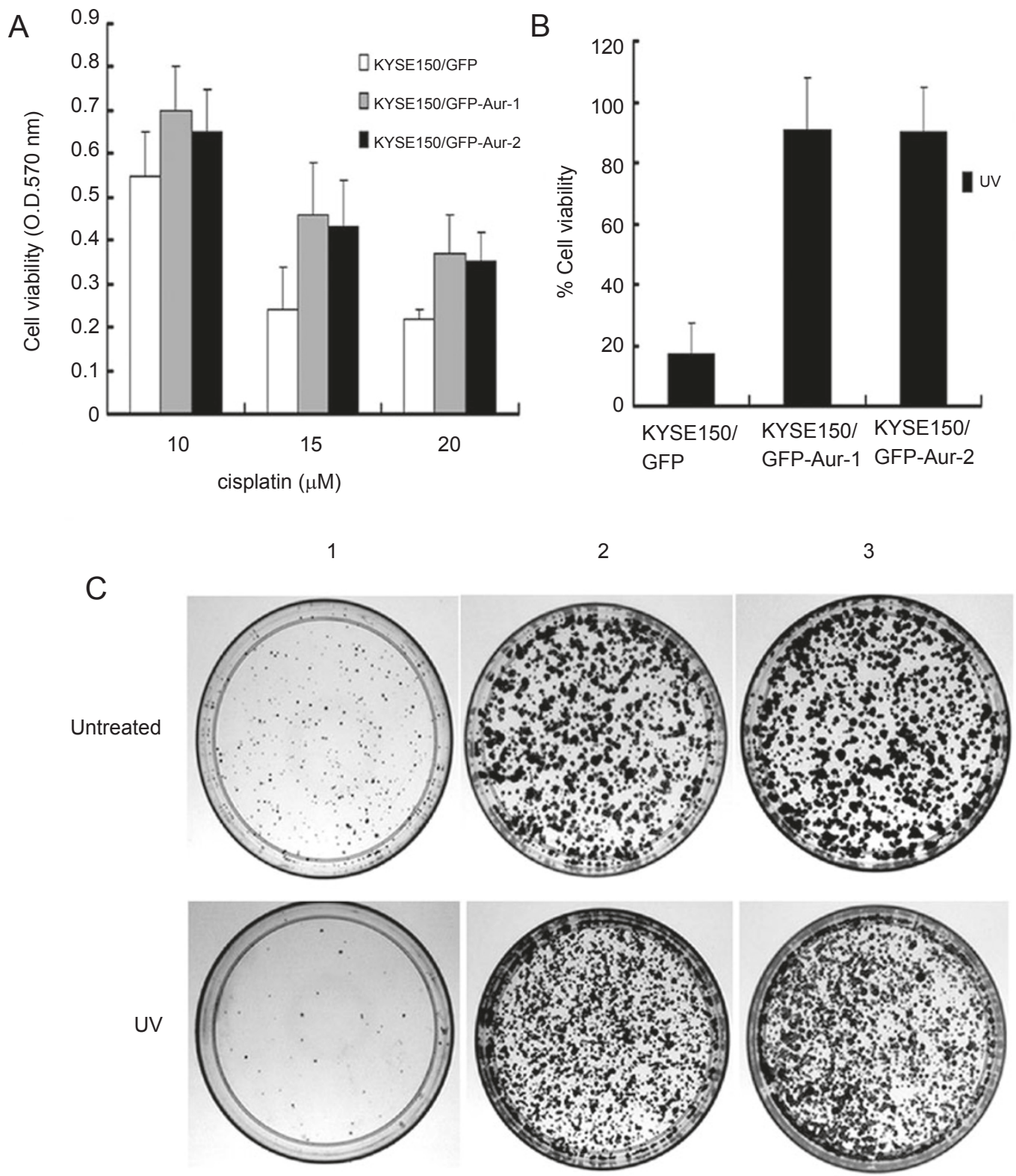

Figure 4 Effect of Aurora-A overexpression on the viability of KYSE150 cells. (A) KYSE150/GFP-Aur and KYSE150/GFP cells were seeded in 96-well plates $\left(5 \times 10^{3}\right.$ cells/well), incubated for $24 \mathrm{~h}$, and then incubated for $72 \mathrm{~h}$ in the presence or absence of increasing cisplatin. Cell viability was determined by the MTT assay. (B-C) KYSE150/GFP-Aur and KYSE150/GFP cells were incubated 10 days after UV irradiation $\left(10 \mathrm{~J} / \mathrm{m}^{2}\right)$ or without irradiation. Cell viability was examined as described in Materials and Methods. (B) Data present the average percentage of colony numbers relative to the untreated cells. All experiments were performed at least three times with consistent and repeatable results. (C) Photographs of crystal violet stained KYSE150/GFP (1), KYSE150/GFP-Aur-1 (2) and KYSE150/GFP-Aur-2 (3) colonies.

was lower than KYSE150/GFP cells. Taken together, cisplatin- and UV-induced apoptosis were significantly inhibited by Aurora-A overexpression.

Overexpression of Aurora-A inhibits caspase-3 and PARP cleavages

We further examined the effect of Aurora-A on cleavages of caspase-3 and PARP. As shown in Figure 6, the noncleaved caspase-3 in KYSE150/GFP cells was much less than that seen in KYSE150/GFP-Aur cells after cisplatin $(10,20,40 \mu \mathrm{M} / \mathrm{L})$ and $\mathrm{UV}\left(10,30,45 \mathrm{~J} / \mathrm{m}^{2}\right)$ treatments, suggesting a strong cleavage of caspase- 3 in KYSE150/ GFP cells, although cleaved caspase- 3 was not detected in the experiments. Moreover, cisplatin and UV treatments 


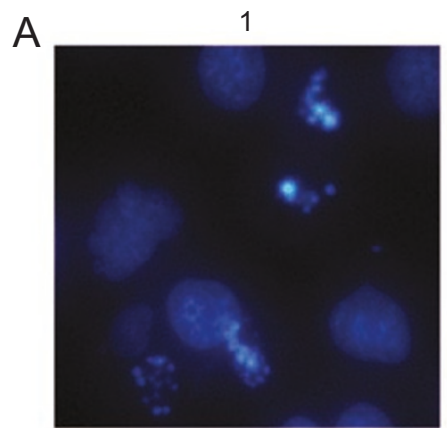

B

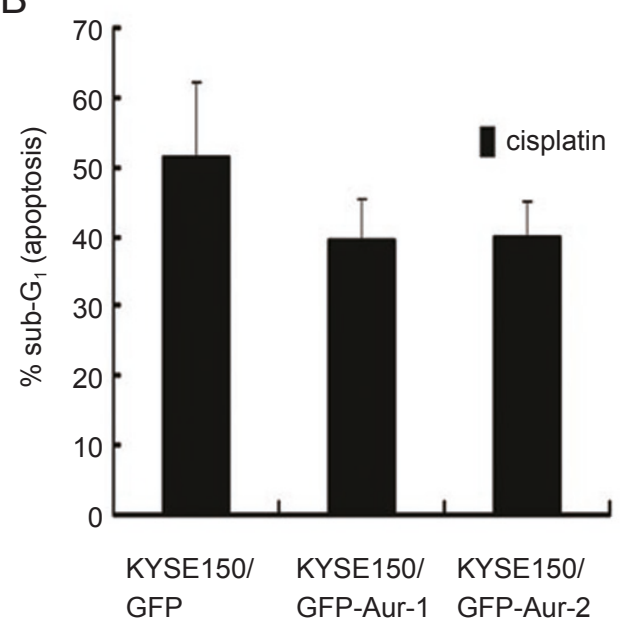

2

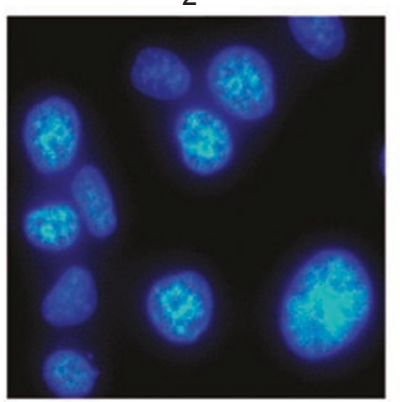

C

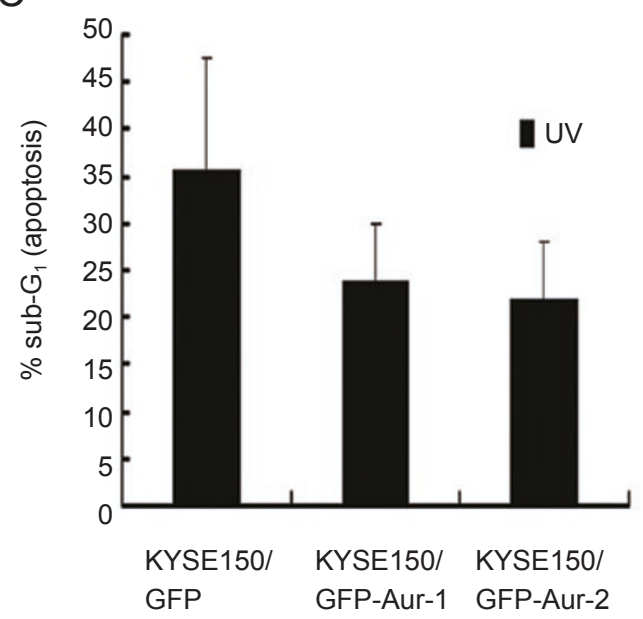

Figure 5 Etfect of Aurora-A overexpression on the apoptosis of KYSE150/GHP (1), KY SE150/GHP-Aur-1 (2) and KYSE150/GFPAur-2 (3). (A) Morphologic changes in cell nuclei visualized by DAPI staining. Cells were incubated for $12 \mathrm{~h}$ after UV (30 J/m²) treatment, and then were observed under fluorescent microscope after DAPI staining. (B-C) Effects of Aurora-A overexpression on sub- $\mathrm{G}_{1}$ cell population. Cells were incubated in a culture medium for $24 \mathrm{~h}$ after cisplatin $(100 \mu \mathrm{M} / \mathrm{L})$ and $\mathrm{UV}\left(45 \mathrm{~J} / \mathrm{m}^{2}\right)$ treatments and analyzed by a flow cytometer. All experiments were performed at least three times with consistent and repeatable results.

resulted in evident PARP cleavage in KYSE150/GFP cells, yielding an $85 \mathrm{kDa}$ fragment. In contrast, cisplatin- or UVinduced PARP cleavage was weaker in KYSE150/GFP-Aur cells. These results indicated that Aurora-A overexpression greatly abrogated the cleavages of caspase- 3 and PARP.

\section{Downregulation of Aurora-A results in increased sensitivity to cisplatin- or UV-induced apoptosis}

To further confirm the role of Aurora-A in resistance to anti-cancer drugs, we examined whether downregulation of Aurora-A correlated with enhanced susceptibility to cisplatin- and UV-induced apoptosis. The endogenous Aurora-A expression in EC9706 cells, which was relatively rich in ESCC cell lines, was downregulated using siRNA strategy. As shown in Figure 7A, Aurora-A protein level dramatically suppressed in EC9706 cells by the siRNA targeting Aurora-A in a dose-dependent manner but not by control siRNA. To assess whether suppression of Aurora-A enhances anti-cancer drugs sensitivity, $50 \mathrm{nM} / \mathrm{L}$ Aurora-A
siRNA- and control siRNA-transfected cells were treated with cisplatin $(5,10,15 \mu \mathrm{M} / \mathrm{L})$ for $72 \mathrm{~h}$ and analyzed with MTT assay. As shown Figure 7B, control siRNA-treated cells displayed higher percentage of viable cells upon cisplatin treatment compared with Aurora-A siRNA-treated cells, which is consistent with our observations that $\mathrm{Au}-$ rora-A overexpressing cells exhibited enhanced cisplatin resistance. Furthermore, downregulation of Aurora-A by Aurora-A-specific siRNA exhibited higher levels apoptosis upon cisplatin and UV treatments compared with control siRNA-transfected cells by percentage of hypodiploid cells (Figure 7C and 7D). These results indicated that siRNAmediated knockdown of Aurora-A can enhance sensitivity of these ESCC cells to anti-cancer drugs.

Overexpression of Aurora-A upregulation of Bcl-2 expression

To further evaluate the mechanism by which Aurora-A overexpression cells were relatively resistant to cisplatin, 


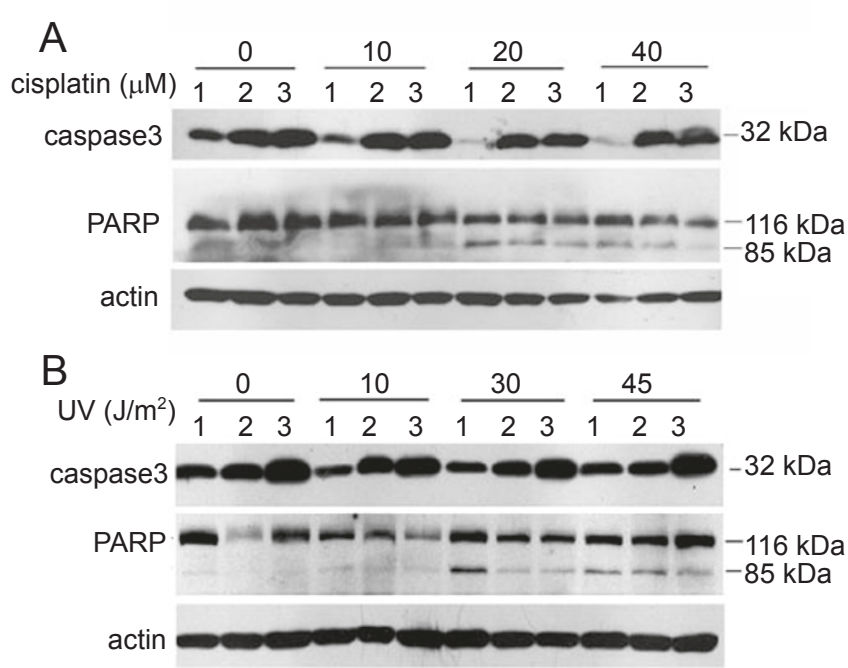

Figure 6 Overexpression of Aurora-A inhibits caspase-3 and PARP cleavages induced by cisplatin (A) and UV treatments (B). KYSE150/ GFP (1), KYSE150/GFP-Aur-1 (2) and KYSE150/GFP-Aur-2 (3) cells were treated with cisplatin $(10,20,40 \mu \mathrm{M} / \mathrm{L})$, UV irradiation $\left(10,30,45 \mathrm{~J} / \mathrm{m}^{2}\right)$ and incubated $12 \mathrm{~h}$. Caspase- 3 and PARP were detected by Western blot analysis. $\beta$-Actin was used as an equal loading control.
UV-induced apoptosis, we examined the expression of apoptosis-related Bcl-2 family member. It was found that expression of Bcl-2 in KYSE150/GFP-Aur cells was higher than KYSE150/GFP cells (Figure 8A). Next we sought to determine whether anti-apoptotic effect of Aurora-A is mediated by its upregulation of Bcl-2. Treatment of KYSE150/GFP-Aur cells with siRNA targeted for $\mathrm{Bcl}-2$ significantly inhibited $\mathrm{Bcl}-2$ protein expression in a dose-dependent manner compared with control siRNA (Figure 8B). Furthermore, control siRNA-treated cells displayed higher percentage of viable cells upon cisplatin treatment compared with Bcl-2 siRNA-treated cells (Figure 8C). Moreover, KYSE150/GFP-Aur cells treated with Bcl-2 siRNA significantly enhanced sub- $\mathrm{G}_{1}$ DNA content upon cisplatin and UV treatments compared with control siRNA-treated cells (Figure 8D and 8E). The results demonstrated that expression of $\mathrm{Bcl}-2$ was upregulated following overexpression of Aurora-A and that the deregulated expression of Bcl-2 (the apoptotic antagonist) contributed to anti-apoptotic effect of AuroraA overexpression.

A

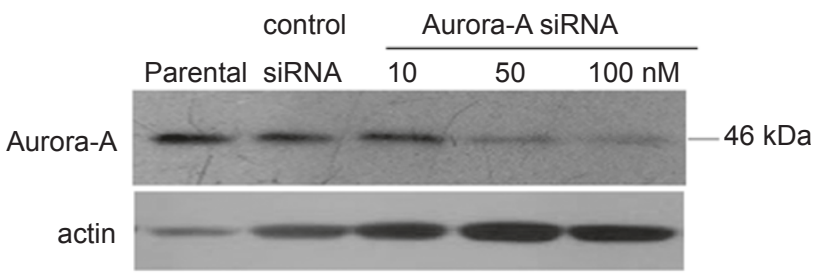

B

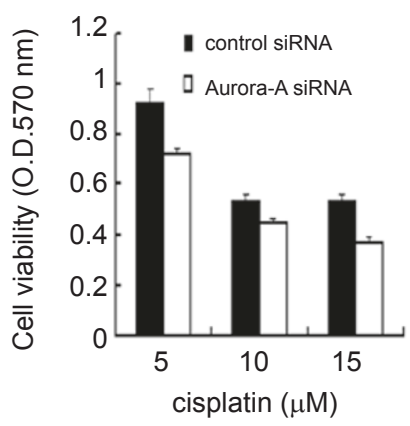

C

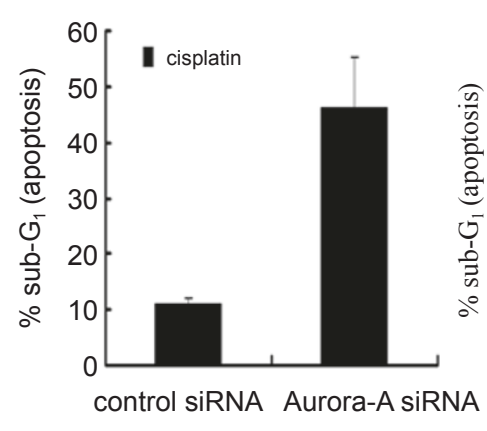

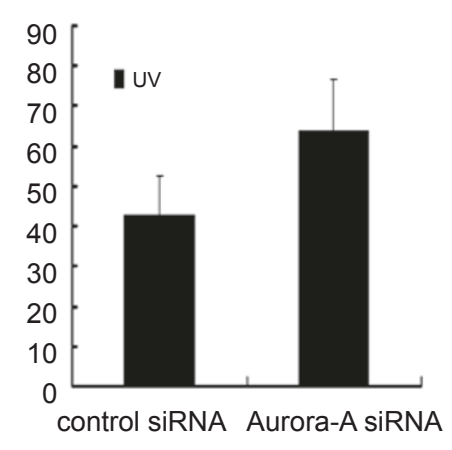

Figure 7 siRNA-directed suppression of Aurora-A enhances sensitivity to cisplatin, UV-induced apoptosis. (A) EC9706 cells were transfected with either Aurora-A siRNA or control siRNA. After $48 \mathrm{~h}$, the cells were collected, and total cellular protein was used for Western blot analysis with anti-Aurora-A antibody as described. $\beta$-Actin was used as an equal loading control. (B) EC9706 cells were transfected with either Aurora-A siRNA or control siRNA. After $24 \mathrm{~h}$, cells were subsequently treated with cisplatin (5, 10, $15 \mu \mathrm{M} / \mathrm{L}$ ) for $72 \mathrm{~h}$, and cell viability was evaluated by MTT assay. (C-D) The sub-G1 DNA content of cells treated with cisplatin $(60 \mu \mathrm{M} / \mathrm{L})$ and $\mathrm{UV}\left(45 \mathrm{~J} / \mathrm{m}^{2}\right)$ was analyzed by flow cytometric analysis. All experiments were performed at least three times with consistent and repeatable results. 

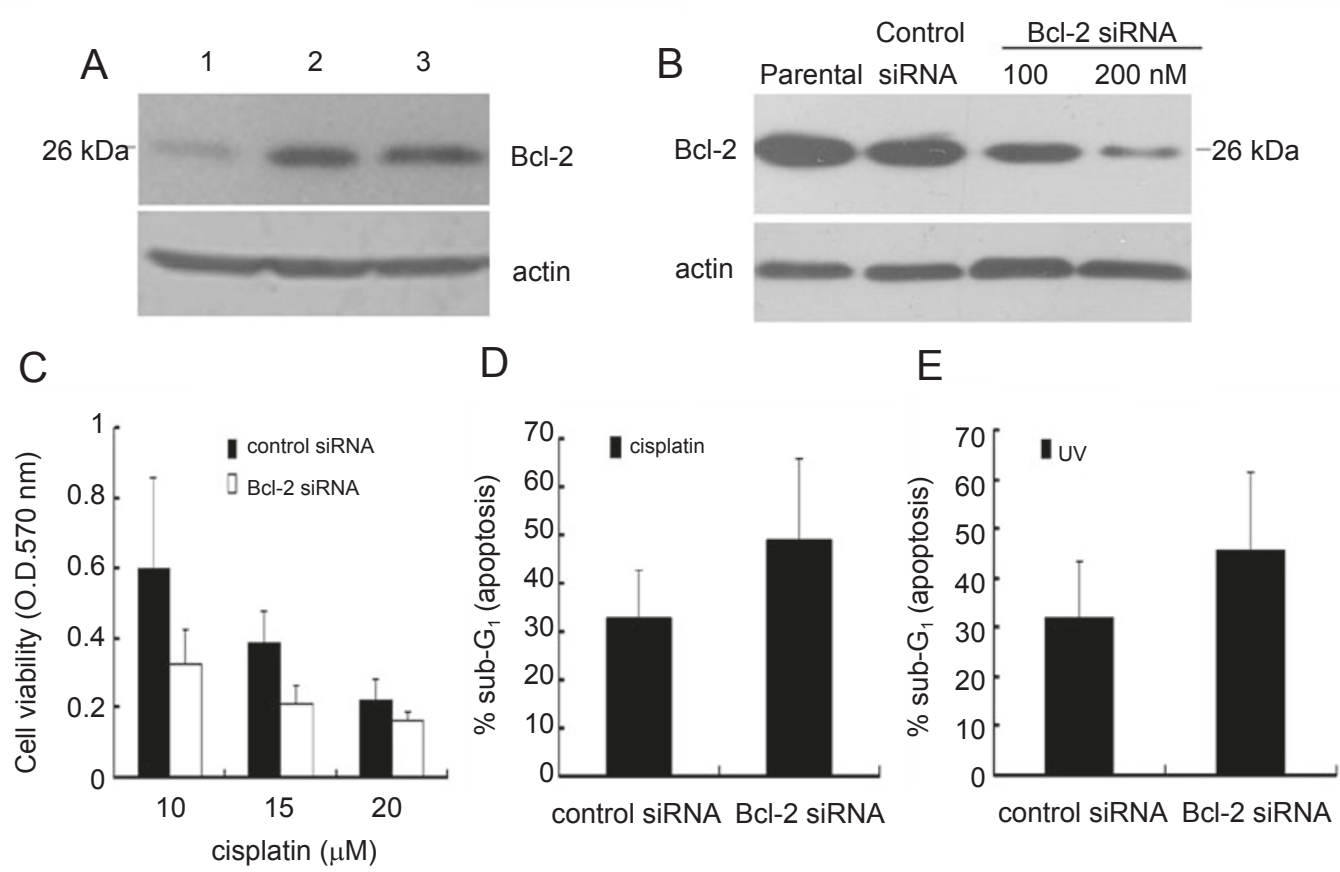

Figure 8 Overexpression of Aurora-A upregulates expression of Bcl-2 and inhibition of Bcl-2 by siRNA abrogates the Aurora-A's survival effect. (A) KYSE150/GFP (Lane 1), KYSE150/GFP-Aur-1 (lane 2) and KYSE150/GFP-Aur-2 (lane 3) cells were collected as described in Materials and Methods. Bcl-2 was detected by Western blot analysis. (B) KYSE150/GFP-Aur cells were transfected with either Bcl-2 siRNA or control siRNA. After $48 \mathrm{~h}$, the cells were collected, and total cellular protein was used for Western blot analysis with anti-Bcl-2 antibody as described. $\beta$-Actin was used as an equal loading control. (C) KYSE150/GFP-Aur cells were transfected with either Bcl-2 siRNA or control siRNA. After $24 \mathrm{~h}$, cells were subsequently treated with $100 \mu \mathrm{M} / \mathrm{L}$ cisplatin for 72 h, and cell viability was evaluated by MTT assay. (D-E) The sub-G1 DNA content of cells treated with cisplatin (100 $\mu$ M/L) and $\mathrm{UV}\left(45 \mathrm{~J} / \mathrm{m}^{2}\right)$ was analyzed by flow cytometric analysis. All experiments were performed at least three times with consistent and repeatable results.

\section{Discussion}

In this study, Aurora-A overexpression results in KYSE150 cells phenotypic alterations including cell proliferation and apoptosis. We demonstrate that Aurora-A overexpression significantly enhances the ability of KYSE150 cells to proliferate and renders cells more resistant to the apoptosis induced by cisplatin and UV. Since many anti-cancer drugs result in DNA cross-linking damage as UV does, the findings in this report are highly of clinical relevance. The results presented in this report describe the inhibitory effects of Aurora-A on apoptosis, which go along with the previous reports [12]. Conversely, siRNA-mediated reduction of Aurora-A expression results in increased sensitivity to cisplatin- and UV-induced apoptosis. Our findings suggest that Aurora-A overxpression can contribute to anti-cancer drugs resistance of ESCC cells. Apoptosis is one of the critical biological factors that correlate with the biological behavior of malignant tumors including cancer progression and poor clinical outcome [14]. Overexpression of Aurora-A kinase has been reported to correlate with ESCC occurrence and progression [10]. Thus, Aurora-A inhibition of drug-induced cell death may not only account for the failure of standard chemotherapy but may also further help explain why Aurora-A overexpression contributes to malignant phenotype of ESCC.

In mammalian cells, two major apoptosis pathways are proposed: the first one involves signals transduced through death receptors; the second relies on a signal from the mitochondria $[15,16]$. Both pathways are involved in an ordered activation of a set of cysteine proteases called caspases [17]. Caspase-3 is a major executioner caspase that is cleaved and activated by the initiator caspase [18]. The active executioner caspases cleave each other and caspase-3 cleaves PARP, which result in caspase-dependent apoptosis $[19,20]$. In this study, we investigated the role of AuroraA in regulating cisplatin- and UV-induced apoptosis. We report that cisplatin and UV can induce apoptosis involving a caspase cascade including cleavages of caspases-3 and PARP in KYSE150 cells. More importantly, we show that overexpression of Aurora-A protects KYSE150 cells from cisplatin, UV-induced apoptosis and inhibits caspase- 3 and 
PARP cleavages. Thus, these results indicate that overexpression of Aurora-A can inhibit cisplatin-, UV-induced apoptosis, probably through interfering with the caspase3/PARP pathway.

$\mathrm{Bcl}-2$ is believed to be primarily involved in suppressing apoptosis and regulating cytochrome c release from mitochondria induced by stimuli, including UV irradiation and many cytotoxic drugs [3]. We report that the expression of Bcl-2 protein increases after tranfection of Aurora-A. Probably, the increased expression of $\mathrm{Bcl}-2$ prevents cytochrome $\mathrm{c}$ release from mitochondria and in turn leads to inactivation of caspase. Conversely, siRNA-mediated reduction of Bcl2 expression abrogates the Aurora-A's effect on inhibiting apoptosis. These data therefore suggest that the inhibitory ability of Aurora-A on cisplatin-, UV-induced apoptosis might be through upregulation $\mathrm{Bcl}-2$ expression. However, the molecular mechanism(s) by which Aurora-A increases expression of Bcl-2 protein remains to be elucidated in the future study. Recently, several targets that are phosphorylated by Aurora-A kinase have been identified, including the tumor suppressor protein p53 and HURP (hepatoma upregulated protein) $[13,21]$. Aurora-A overexpression leads to increased degradation of p53 and stabilization of HURP, causing facilitating oncogenic transformation of cells. Therefore, it is speculated that one of possibilities might be that Aurora-A is able to phosphorylate Bcl-2 protein and in turn stabilizes this protein.

In summary, these data provide interesting evidence that the molecular events involved in overexpression of Aurora-A inhibition of human ESCC cells apoptosis. Overexpression of Aurora-A can contribute to anti-cancer drugs resistance of ESCC cells. Further, Aurora-A inhibition of cisplatin- and UV irradiation-induced apoptosis, suggesting a novel mechanism that is closely related to Aurora-A-induced tumorigenesis and progression. We have demonstrated that Aurora-A knockdown may increase the sensitivity of cisplatin and UV induced apoptosis in ESCC cells. The siRNA oligonucleotides used in this study are potent tools for modulating the Aurora-A gene expression and they may be developed into attractive antitumor therapeutics. Therefore, further understanding of the signaling pathways that Aurora-A controls apoptosis in the human ESCC will help with the discovery of novel targeted agents, and may lead to the development of new approaches for effective therapy.

\section{Acknowledgements}

This work was supported by the National Natural Science Foundation (30225018) and National Key Basic Research Program of China (2002CB513101). We thank Dr Shimada (Kyoto University) for providing KYSE esophageal carcinoma cell lines.

\section{References}

1 Lin YC, Wu MY, Li DR, Wu XY. Zheng RM, Prognostic and clinicopathological features of E-cadherin, alpha-catenin, betacatenin, gamma-catenin and cyclin D1 expression in human esophageal squamous cell carcinoma. World J Gastroenterol 2004; 10:3235-3239.

2 Toshimitsu H, Hashimoto K, Tangoku A, et al. Molecular signature linked to acquired resistance to cisplatin in esophageal cancer cells. Cancer Lett 2004; 211:69-78.

3 Sun SY, Yue P, Zhou JY, et al. Overexpression of BCL-2 blocks TNF-related apoptosis-inducing ligand (TRAIL)-induced apoptosis in human lung cancer cells. Biochem Biophys Res Commun 2001; 280:788-797.

4 Sjostrom J, Bergh J. How apoptosis is regulated, and what goes wrong in cancer. BMJ 2001; 322:1538-1539.

5 Liu Q, Kaneko S, Yang L, et al. Aurora-A abrogation of p53 DNA binding and transactivation activity by phosphorylation of serine 215. J Biol Chem 2004; 279:52175-52182.

6 Sen S, Zhou H, White RA. A putative serine/threonine kinase encoding gene BTAK on chromosome $20 \mathrm{q} 13$ is amplified and overexpressed in human breast cancer cell lines. Oncogene 1997; 14:2195-2200.

7 Bischoff JR, Anderson L, Zhu Y, et al. A homologue of Drosophila aurora kinase is oncogenic and amplified in human colorectal cancers. EMBO J 1998; 17:3052-3065.

8 Li D, Zhu J, Firozi PF, et al. Overexpression of oncogenic STK15/BTAK/Aurora A kinase in human pancreatic cancer. Clin Cancer Res 2003; 9:991-997.

9 Tanaka E, Hashimoto Y, Ito T, et al. The clinical significance of Aurora-A/STK15/BTAK expression in human esophageal squamous cell carcinoma. Clin Cancer Res 2005; 11:1827-1834.

10 Tong T, Zhong Y, Kong J, et al. Overexpression of Aurora-A contributes to malignant development of human esophageal squamous cell carcinoma. Clin Cancer Res 2004; 10:7304-7310.

11 Goepfert TM, Adigun YE, Zhong L, et al. Centrosome amplification and overexpression of aurora A are early events in rat mammary carcinogenesis. Cancer Res 2002; 62:4115-4122.

12 Anand S, Penrhyn-Lowe S, Venkitaraman AR. AURORA-A amplification overrides the mitotic spindle assembly checkpoint, inducing resistance to Taxol. Cancer Cell 2003; 3:51-62.

13 Katayama H, Sasai K, Kawai H, et al. Phosphorylation by aurora kinase A induces Mdm2-mediated destabilization and inhibition of p53. Nat Genet 2004; 36:55-62.

14 Noguchi T, Takeno S, Shibata T, et al. Nuclear BAG-1 expression is a biomarker of poor prognosis in esophageal squamous cell carcinoma. Dis Esophagus 2003; 16:107-111.

15 Cryns V, Yuan J. Proteases to die for. Genes Dev 1998; 12:15511570 .

16 Green DR. Apoptotic pathways: the roads to ruin. Cell 1998; 94:695-698.

17 Alnemri ES, Livingston DJ, Nicholson DW, et al. Human ICE/ CED-3 protease nomenclature. Cell 1996; 87:171

18 Igney FH, Krammer PH. Death and anti-death: tumour resistance to apoptosis. Nat Rev Cancer 2002; 2:277-288.

19 Rathmell JC, Thompson CB. The central effectors of cell death in the immune system. Annu Rev Immunol 1999; 17:781-828. 
20 Boulares AH, Yakovlev AG, Ivanova V, et al. Role of poly (ADPribose) polymerase (PARP) cleavage in apoptosis. Caspase 3resistant PARP mutant increases rates of apoptosis in transfected cells, J Biol Chem 1999; 274:22932-22940.
21 Yu CT, Hsu JM, Lee YC, et al. Phosphorylation and stabilization of HURP by Aurora-A: implication of HURP as a transforming target of Aurora-A. Mol Cell Biol 2005; 25:5789-5800.

Edited by Anning Lin 